UB-ECM-PF 92/1

January 1992

\title{
Spectrum of Relativistic Fermions in a 2d Doped Lattice
}

\author{
D. Espriu and J. MATiAs \\ D.E.C.M. \\ Universitat de Barcelona \\ Diagonal, 647 \\ E-08028 Barcelona
}

\begin{abstract}
Motivated by some previous work on fermions on random lattices and by suggestions that impurities could trigger parity breaking in $2 \mathrm{~d}$ crystals, we have analyzed the spectrum of the Dirac equation on a two dimensional square lattice where sites have been removed randomly - a doped lattice. We have found that the system is well described by a sine-Gordon action. The solitons of this model are the lattice fermions, which pick a quartic interaction due to the doping and become Thirring fermions. They also get an effective mass different from the lagrangian mass. The system seems to exhibit spontaneous symmetry breaking, exactly as it happens for a randomly triangulated lattice. The associated "Goldstone boson" is the sine-Gordon scalar. We argue, however, that the peculiar behaviour of $\langle\bar{\psi} \psi\rangle$ is due to finite size effects.
\end{abstract}


Sometime ago the spectrum of the Dirac equation on a two dimensional random lattice was studied in great detail[1]. It was then found numerically that the density of eigenvalues $\rho(\lambda)$ on randomly triangulated lattices exhibits a rather peculiar behaviour. Instead of behaving linearly for small values of the eigenvalue $\lambda, \rho(\lambda) \sim \lambda$, as it does in the continuum and also in a regular lattice (albeit with an additional factor of 4 due to doubling), the density of eigenvalues does not vanish as $\lambda \rightarrow 0$ for the lattices considered. Indeed it would seem that, as the lattice size increased, $\rho(\lambda)$ would go to a constant value or even diverge as $\lambda \rightarrow 0$. Accordingly, the fermion condensate, which on general grounds is related to the spectral density

$$
\langle\bar{\psi} \psi\rangle=\pi \rho(0)
$$

would not vanish. Since we are in a two dimensional system which is not supposed to undergo spontaneous symmetry breaking[2], the behaviour of $\langle\bar{\psi} \psi\rangle$ in a random lattice is quite unexpected. Of course from the numerical evidence alone one cannot rigorously conclude that

$$
\lim _{m \rightarrow 0} \lim _{V \rightarrow \infty}\langle\bar{\psi} \psi\rangle \neq 0
$$

since one is forced to work with finite lattices, but the results of [1] strongly suggest that in the limit $(2)\langle\bar{\psi} \psi\rangle$ tends to a finite constant. This non-zero value for $\langle\bar{\psi} \psi\rangle$ is clearly due to the "interactions" induced by the randomness on the otherwise free fermions.

It was also observed in [1] that the pseudoscalar Green function $\left\langle\left(\bar{\psi} \gamma^{5} \psi\right)(x)\left(\bar{\psi} \gamma^{5} \psi\right)(0)\right\rangle$ has, in addition to a pole at twice the mass of the fermion, an unmistakeable signal of another particle in the spectrum with a mass $m_{55} \sim \sqrt{m / a}$. The fermion mass itself is not affected by the randomness in any noticeable way. The extra particle seen in the pseudoscalar channel would correspond to the (pseudo) "Goldstone boson" of the broken $U(1)_{A}$ symmetry. The reason for the quotation marks is that no genuine Goldstone boson is supposed to exist in $2 \mathrm{~d}$ due to the infrared problems characteristic of $2 \mathrm{~d}$ physics. However, we never take $m \rightarrow 0$ in the random lattice because we always work with finite volumes. In those conditions the particle found in [1] behaves as a pseudo Goldstone boson, so the question of whether, in the appropriate limits, the Goldstone goes away or not is somewhat academic. The fact is that physics on a $2 \mathrm{~d}$ finite random lattice is very well accounted for by assuming that there is spontaneous symmetry breaking, with all its consequences. The particle found in [1] is, however, unstable; it decays into two fermions.

The other remarkable feature of the random lattice is that the doubled fermions go away, at least for the free theory[1,3]. Indeed, the correlators on the random lattice behave at long distances exactly as their continuum counterparts, normalization included. The doubles have acquired a very large dynamically generated mass and have disappeared from the spectrum. The only trace of the would be doubles is the "Goldstone boson" discussed 
in the previous paragraph. The anomaly is also well reproduced. This is possible because the random lattice bypasses one of the hypothesis of the Nielsen-Ninomiya theorem[4]. In practice, for interacting theories some mass fine tuning turns out to be necessary in a way somewhat similar to what has to be done for Wilson fermions[3]. One's hope is that some of the features observed in [1] (in particular the appearance of $\langle\bar{\psi} \psi\rangle \neq 0$ ) must be, in some sense, universal. Relativistic fermions on a regular two dimensional doped lattice is a natural, more tractable, alternative.

There is also another reason to study relativistic fermions in a doped lattice. Many interesting materials in condensed matter physics (in particular those where high $T_{c}$ superconductivity has been described) are two dimensional in nature. One could well think of a regime in which some quenched impurities prevent electrons (or holes) from occupying some lattice sites. If we mimic this potential by some repulsive potential the corresponding Schrödinger equation would be

$$
i \frac{\partial}{\partial t} \phi=-\frac{1}{2 m} \Delta^{2} \phi+V \phi
$$

where $\Delta$ is the lattice laplacian and $V$ vanishes if the site is not occupied and is large and positive if there's an impurity in the site in question. We will not consider the relevant case of having a finite electron density, neither interactions amongst them. It turns out that the physical situation described by (3) can be obtained as the non-relativistic limit of the Dirac equation

$$
i \frac{\partial}{\partial t} \psi(x, t)=-i \alpha_{i} \Delta_{i} \psi(x, t)+m \sigma_{3} \omega(x) \psi(x, t)
$$

provided that we let $w(x) \rightarrow \infty$ at the impurity sites. $\Delta_{i}$ is the lattice derivative in the $i$ direction, while the $t$ direction is kept continuous. The eigenstates and eigenvalues of the 2 dimensional Dirac equation correspond to the energy eigenvalues and eigenstates of the $3 \mathrm{~d}$ quantum hamiltonian. This problem is exactly equivalent to a lattice where the impurity sites have been removed. In order to see this we simply have to scale the fields to absorb $w(x)$. The resulting lattice Dirac operator will be

$$
D(x, y)=\frac{\gamma_{\mu} l_{\mu}(x, y) U(x, y)}{2 \sqrt{w(x) w(y)}}+m \delta(x, y)
$$

where $l_{\mu}(x, y)$ are the lattice vectors and $U(x, y)$ is a gauge field that we have added for later use. The only difference between the spectrum obtained by finding the eigenvalues of (5) and then going to the non-relativistic limit and the Schrödinger problem described by (3) would be that squaring $D$ produces a laplacian connecting sites at distance two. This is of course well known and it just tells us that we will have four species. In contrast to a random lattice, doping a regular lattice is not enough to remove the doubling. 
In what follows we will see that free fermions in a two dimensional doped lattice exhibit, even for small masses and large volumes, a large value for $\langle\bar{\psi} \psi\rangle$, a phenomenon closely resembling the one taking place in a random lattice. We will also see that there is a pseudo Goldstone boson too. As we pointed out before, both observations are clearly at odds with the common lore. However, the numerical results look very convincing: a person who has never heard about Mermin-Wagner's or Coleman's theorem would conclude that indeed there is spontaneous symmetry breaking in this $2 \mathrm{~d}$ system. Yet, we have reasons to believe that the appearance of spontaneous symmetry breaking in the doped lattice is, rather paradoxically, a finite size effect. As we will show, the complete system, fermions plus Goldstone boson, is well described for any value of the mass by a massive Thirring model[5] and its bosonic counterpart, the sine-Gordon model[6]. The Thirring fermions will correspond to our original lattice fermions, the doping having induced a quartic chiral invariant interaction. Using this information we will see that the symmetry breaking disappears in the infinite volume limit. We will also see that there are more differences between a random lattice and a doped lattice than we originally thought.

Our system consists of a two dimensional square regular lattice with lattice spacing $a$ where we have taken out randomly points of the space, to simulate impurities in a crystal. The lattice is basically a regular square lattice where pairs of points separated a distance $a$ are joined by a link if none of the points of the couple is an impurity and otherwhise there is no link. The construction of the dual lattice is straightforward except for the dual cells associated to the points near an impurity which have to be modified to make sure the sum of areas is equal to the complete volum of the system. There is some freedom in doing this which does not seem to matter. The symmetries of the problem $(C$, charge conjugation, and chirality, $\gamma_{5}$ ) tell us that the eigenvalues come in sets of four. Parity and discrete rotations are not exact symmetries any longer. They have been broken by the doping; their restoration in an broader statistical sense has to be checked explicitly (they are indeed restored).

Let us begin by evaluating the chiral condensate in our doped lattice. The condensate is defined by

$$
\langle\bar{\psi} \psi\rangle=\frac{1}{V} \sum_{x=1}^{V} \omega(x) \operatorname{tr}(D+m)^{-1}(x, x)=\frac{1}{V} \sum_{\lambda \geq 0} \frac{2 m}{\lambda^{2}+m^{2}}
$$

We have computed this expression using both periodic and antiperiodic boundary conditions for the fermion fields without finding any noticeable differences. We have considered several lattice sizes and different densities of impurities. In all the cases we have examined $\langle\bar{\psi} \psi\rangle$ is much bigger than in the regular lattice case, showing a behaviour which is not too different from the random lattice. This is illustrated in Fig. 1. The behaviour of $\langle\bar{\psi} \psi\rangle$ 
strongly suggests that the $U(1)_{A}$ symmetry of free fermions is spontaneously broken by the onset of randomness. The breaking appears to be stronger for higher density of impurities. For smallish sizes $\langle\bar{\psi} \psi\rangle$ fluctuates rather wildly from a given lattice to another, but beyond some size the curves order nicely. Notice that for a given lagrangian mass $\langle\bar{\psi} \psi\rangle$ seems to decrease slowly with the volume.

Has the fermion mass been somehow modified by the doping? The zero momentum fermion propagator will give this information to us. It is defined by

$$
G^{\alpha \beta}(x)=\frac{1}{V} \int d x_{1} d y_{1} d x_{2} d y_{2}\left\langle\bar{\psi}^{\beta}\left(x_{1}, y_{1}\right) \psi^{\alpha}\left(x_{2}, y_{2}\right)\right\rangle \delta\left(x-\left(x_{1}-x_{2}\right)\right)
$$

where we have averaged over vertical bins of width $a / 2$ or slightly greater if there is an impurity on the left or right side of the bin we are averaging. Using symmetry arguments we can write $G(x)=A(x) 1+B(x) \gamma_{1}$, so $A(x)+B(x)$ describes a particle propagating forward in time. In a regular lattice of size $V=L_{1} \times L_{2}$

$$
A(x)+B(x)=\frac{1}{L_{1}} \sum_{n=1}^{L_{1}} \frac{\sin p \sin p x+M a \cos p x}{(M a)^{2}+\sin ^{2} p} \quad p=\frac{2 \pi n}{L_{1}}
$$

(We have mostly used periodic boundary conditions for these fits.) Comparing this expression with the curve obtained numerically by inverting the Dirac operator one obtains the mass of the propagating particle. The fits are always extremely good, except for the smaller masses (typically below $m=0.05$ ) where performing a quenched average over a set of lattices may be required. We have found that, unlike in a random lattice, the fermion mass observed in the propagator, $M$, is no longer the lagrangian mass $m$. The propagator mass grows linearly with the lagrangian mass for large masses, but with a coefficient larger than one. This coefficient, in addition, increases with the doping but seems to be volume independent. For small masses, on the other hand, there is some saturation at an almost constant value, as can be observed in Fig. 2, suggesting that in the $m \rightarrow 0$ limit the propagator mass $M(m)$ stays finite. The finite value to what $M$ tends as $m \rightarrow 0, M(0)$, clearly decreases with increasing volumes, indicating that it may be due to finite size effects. $M(0)$ grows with the density of impurities. In addition to a mass renormalization induced by the doping, there is also some wave function renormalization. The mass and wave function corrections obtained from the fermion propagator coincide very nicely with the ones that come from the connected part of the scalar propagator $P_{11}(x)=\langle(\bar{\psi} \psi)(x)(\bar{\psi} \psi)(0)\rangle$. In the absence of other particles in this channel this is expected to decay as $2 M$ and it does. The agreement is really good, as Fig. 3 shows. From measuring this Green function we also find out that the doubles subsist, the overall normalization of $P_{11}$ being approximately four times the naive one. In some sense our system resembles a random lattice but it is still ordered enough to allow the survival of the doubles. 
Is there a pseudo "Goldstone boson" ? Let's examine the pseudoscalar propagator

$$
P_{55}(x)=\frac{1}{V} \int d x_{1} d y_{1} d x_{2} d y_{2}\left\langle\left(\bar{\psi} \gamma^{5} \psi\right)\left(x_{1}, y_{1}\right)\left(\bar{\psi} \gamma^{5} \psi\right)\left(x_{2}, y_{2}\right)\right\rangle \delta\left(x-\left(x_{1}-x_{2}\right)\right)
$$

To calculate this correlator we invert the Dirac operator, using periodic boundary conditions, for different masses and densities. By plotting this zero momentum propagator we clearly see the contribution from two type of particles. First of all, there is an exponential decay (actually a cosh behaviour in our finite lattice) with a mass $2 M$, exactly as for the $P_{11}$ propagator. This corresponds to the free propagation of the fermion - antifermion pair in the lattice and it is described by an expression of the form

$$
P_{55}^{r e g}(x)=C \frac{2}{L_{1}^{2} L_{2}} \sum_{n=1}^{L_{1}} \sum_{m=1}^{L_{1}} \sum_{k=1}^{L_{2}} \frac{\left((M a)^{2}+\sin p_{1} \sin p_{2}+\sin ^{2} q\right) \cos \left(p_{1}-p_{2}\right) x}{\left((M a)^{2}+\sin ^{2} p_{1}+\sin ^{2} q\right)\left((M a)^{2}+\sin ^{2} p_{2}+\sin ^{2} q\right)}
$$

where $p_{1}=2 \pi n / L_{1}, p_{2}=2 \pi m / L_{1}, q=2 \pi k / L_{2}$ and $C$ is the additional wave function renormalization factor we mentioned before. There are no free parameters here, everything is obtained from the fermion propagator. This would be the only contribution in a regular lattice (but with $M=m$ ). However, once this behaviour has been subtracted from the measured propagator there is still a clear signal of a particle in the pseudoscalar channel. This signal is perfectly adjusted by the propagator of a scalar particle in a finite box, with a coefficient that we fit. The fit to the scalar particle, with mass $m_{55}$, plus free fermions, with mass $M$, really works perfectly (Fig. 3) for all the desities of impurities we have tried (up to 20\%) and for the whole range of sizes.

The above situation is again reminiscent of the random lattice. However, here the scalar particle, that we interpret as the pseudo "Goldstone boson" of the "broken" symmetry, is actually the lighter state in its channel and therefore stable, $m_{55}<2 M$. Its mass $m_{55}$ as a function of the lagrangian mass $m$ is plotted in Fig. 4. For large values of $m$, $m_{55}$ grows linearly with $m$. For smaller values there are deviations with respect to this behaviour. We have found that $m_{55}$ is extremely well adjusted by the formula

$$
m_{55}^{2}=c_{1} m^{b}+c_{2} m^{2} \quad, \quad b<1
$$

In the random lattice, on the contrary, the behaviour is $m_{55}^{2} \sim m$ to a very good approximation. Very small values of the mass were not investigated in [1].

Let us now try to gain some theoretical understanding of the above results. We have seen that $\langle\bar{\psi} \psi\rangle$ is large in our system. On general grounds we then expect that the generator of the broken symmetry acting on the vacuum state produces a Goldstone-like particle and indeed this particle is clearly seen in the pseudoscalar channel. Should the symmetry be 
exact, the "Goldstone" would be exactly massless. However there is a explicit breaking of the form

$$
m \bar{\psi}_{R} \psi_{L}+m \bar{\psi}_{L} \psi_{R}
$$

The bosonization rules in two dimensions force us to make the identifications

$$
\bar{\psi}_{L} \psi_{R} \sim e^{-i \frac{\sqrt{2}}{f} \phi} \quad \bar{\psi}_{L} \psi_{R} \sim e^{i \frac{\sqrt{2}}{f} \phi}
$$

On general grounds we expect for this particle a lagrangian

$$
\mathcal{L}=\frac{1}{2}\left(\partial_{\mu} \phi\right)^{2}+\hat{m}^{2} \frac{f^{2}}{2}: \cos \left(\frac{\sqrt{2}}{f} \phi\right):
$$

The sign of the second term is irrelevant; it can be absorbed in a shift of $\phi$. The constants $\hat{m}$ and $f$ cannot be determined by symmetry arguments alone. We know, however, that $\hat{m}$ must vanish when $m \rightarrow 0$. It is also easy to see that for free fermions $f^{2}=1 / 2 \pi$. Any departure from this value will indicate that the fermions are not free. This will indeed be the case for non-zero doping density.

Eq. 14 is of course nothing but the well known sine-Gordon lagrangian. Sine-Gordon is solvable at the classical level and its quantum spectrum can be found using the WKB method[8] and from other considerations[9]. The exact spectrum consists of two type of particles: solitons and antisolitons, with a mass $M_{S}=8 \hat{m} / \gamma$, and a finite number of soliton-antisoliton bound states with masses $M_{n}=(16 \hat{m} / \gamma) \sin (n \gamma / 16)$ where $n=$ $1,2,3, . .<(8 \pi / \gamma)$ and $\gamma=\frac{2}{f^{2}}\left(1-\frac{1}{4 \pi f^{2}}\right)^{-1}$. The solitons of this model are the fermions of the Thirring model, which as Coleman demonstrated[7] long ago has the same spectrum as sine-Gordon, and whose lagrangian is

$$
\mathcal{L}=\bar{\psi} \gamma_{\mu} \partial_{\mu} \psi+M_{0} \bar{\psi} \psi-\frac{g}{2} \bar{\psi} \gamma_{\mu} \psi \bar{\psi} \gamma_{\mu} \psi
$$

The physical mass of the fermions, renormalized by the quartic interactions, is $M_{S}$. The relation between the quartic Thirring coupling $g$ and $f$ is $g / \pi=2 \pi f^{2}-1$.

One may worry to what extent the equivalence Thirring - sine-Gordon holds on a lattice. Quite apart from general symmetry arguments, let us recall that the equivalence is seen by perturbing both theories around the massless case and comparing Green functions[7]. It is easy to see that this equivalence holds on a lattice too. The lattice massless propagators corresponding to the operators $\bar{\psi} \psi$ in the l.h.s of (13) are, in the infinite volume limit, identical to the ones corresponding to their bosonic counterparts in the r.h.s. of (13), but defined in a lattice with spacing $2 a$. We expect to have four of such bosons. We have seen that the scalar particle in the $P_{55}$ channel can be described by the sineGordon lagrangian. It is then natural to identify the Thirring fermions with our doped 
lattice fermions, whose bound state in sine-Gordon (fermion-antifermion bound state in Thirring) corresponds to our Goldstone boson. The Thirring - sine-Gordon correspondence predicts the mass ratio

$$
\frac{m_{55}}{2 M}=\sin \frac{n \gamma}{16}
$$

Indeed, for all densities we always find this ratio to be less than one. Note that since $\hat{m}$ contains some normal ordering renormalization, $M_{S}$ and $M_{n}$ cannot be unambiguosly determined, but the ratio is well defined. We only observe one single pole in the $P_{55}$ channel, so it must be the case that $\gamma>4 \pi$ (or, equivalently, $f^{2}<3 / 4 \pi$ ). When comparing the different spectra it should be borne in mind that finite size effects affect Thirring and sine-Gordon differently. These effects are expected to be smallest for relatively large masses. Indeed we observe that the ratio (16) is very approximately constant beyond some mass and from this ratio we can extract the parameter $f$ (or $g$ ) that characterizes the model. For the doping densities $20,10,4.9 \%$ we get $g=1.06,0.90,0.83$, respectively. Not surprisingly, the value of $g$ increases with the doping concentration. In the $m \rightarrow 0$ limit, the mass of the Thirring fermion is substantially modified by finite size effects[10]. In the zero mass limit one expects $M \sim g^{2} / L$. This is in good agreement with the "saturation" behaviour observed in Fig. 2. The fermion-antifermion composite boson, on the other hand, becomes free in that limit so we expect $m_{55} \rightarrow 0$ as $m \rightarrow 0$ even in a finite volume.

Contrary to the doped case, the random lattice has $m_{55}>2 M$. This implies that the random lattice falls in a domain of parameters where the correspondence sine-Gordon - Thirring fails. (The effective potential of sine-Gordon is unbounded from below for $f^{2}<1 / 4 \pi$.) Indeed, in the random lattice the "Goldstone" decays into a pair of physical fermions with mass $m$. Since in a random lattice the correspondence with Thirring fails, we have no reason to expect a four fermion term. This was confirmed in [1]; the physical fermions are indeed free on a random lattice. The doubles, however, have acquired a mass of $\mathcal{O}(1 / a)$ due to the randomness. Another difference we have between the random lattice case and our doped lattice is the behaviour of $m_{55}$ as a function of the mass. While we find a behaviour of the form (11), in the random lattice a dependence of the form $m_{55} \sim \sqrt{m}$ describes well the data. All this suggests that the randomly triangulated lattice exhibits, for a broad range of masses, a behaviour much more similar to the one of a system undergoing genuine spontaneous symmetry breaking.

Now we would like to turn to $\langle\bar{\psi} \psi\rangle$ again. We have arrived at the conclusion that our doped lattice is well described by Thirring / sine-Gordon. Since the partition function of the latter can be computed in the WKB approximation, by making a derivative with respect to $m$ we can estimate theoretically the contribution from the bosonic sector of the theory to $\langle\bar{\psi} \psi\rangle$. Adding the contribution from the solitonic sector - just a loop of fermions with mass $M$ - we can compute $\langle\bar{\psi} \psi\rangle$. We will restrict ourselves to small masses, where 
the use of a continuum formalism for a scalar is justified. For masses $m$ comparable to the lattice spacing $a$ one would have to estimate the partition function using consistently the lattice action. After evaluating the partition function by the saddle point method and taking care of the quadratic fluctuations around a doublet state $[8,11]$, one arrives at the following result for the partition function in a box of size $L \times T$ in Minkowski space

$$
\operatorname{Tr} e^{i H T} \simeq L T \sum_{n} \sum_{r} \sqrt{\frac{M_{r}}{\left(T^{2}-(n L)^{2}\right)^{3 / 2}}} \exp \left(-i M_{r} \sqrt{T^{2}-(n L)^{2}}\right)
$$

The sum over $r$ runs over all doublet states. As we have seen we have only one. The above expression is remarkably simple. Apart from some prefactors this is the first quantized action for a relativistic scalar particle in a one-dimensional box of size $L$, if we take into account that $n L / T$ is the velocity $v$ of the particle (which may wrap around several times). $v$ (or $n$ ) is in fact a collective coordinate. In euclidean space time we have to Wick rotate the coordinates appropriately. We also have to include solutions that wrap in the $T$ direction, to restore the $L \leftrightarrow T$ symmetry. So, finally

$$
Z_{S G}=\operatorname{Tr} e^{-H T} \simeq L T \sum_{n} \sum_{m} \sqrt{\frac{m_{55}}{\left((m T)^{2}+(n L)^{2}\right)^{3 / 2}}} \exp \left(-m_{55} \sqrt{(m T)^{2}+(n L)^{2}}\right)
$$

The value of $\langle\bar{\psi} \psi\rangle$ is obtained as

$$
\langle\bar{\psi} \psi\rangle=-\frac{1}{L T} \frac{\partial}{\partial m} \log Z
$$

For the sizes and masses we have been considering, the leading contribution to the chiral condensate is, for a square lattice,

$$
\langle\bar{\psi} \psi\rangle \simeq 4 \times \frac{1}{2 L} \frac{\partial m_{55}}{\partial m}
$$

where we have included the factor of 4 due to doubling. For small masses $m_{55} \sim m^{b / 2}$, with $b<1$, so the chiral condensate behaves as $1 / L m^{(1-b / 2)}$. This expression clearly shows that the appearance of $\langle\bar{\psi} \psi\rangle \neq 0$ in a doped lattice is a finite size effect, at least for the contribution that comes from the "Goldstone boson". What about the contribution from the solitonic sector? This is proportional to $M \log M$, and since $M$ vanishes in the infinite volume limit, it will vanish too. Fig.1c shows the comparison between the values of $\langle\bar{\psi} \psi\rangle$ obtained by numerical evaluation in the doped lattice and analytically from Thirring / sine-Gordon in the WKB approximation. The agreement is qualitatively good.

To conclude we would like to mention several other points related to our system.

i) Since our doped lattice has doubled fermions one expects the chiral anomaly to vanish. That this is the case it can be seen by switching on the gauge field $U(x, y)$ and 
making a perturbative expansion. The first order contribution would require the evaluation of the Green function $\left\langle\left(\bar{\psi} \gamma^{5} \psi\right)(0)\left(\bar{\psi} \gamma_{\mu} \gamma^{5} \psi\right)(x)\right\rangle$, which is precisely the one leading to the anomaly in two dimensions. It can be seen easily that this quantity is identically zero.

ii) A natural question to ask is whether the presence of randomness has induced localization of some sort. The answer is no. We have checked that all the eigenvectors of the Dirac equation are extended states, as far as one can tell numerically. This is also at variance with the situation in a randomly triangulated lattice where it was seen that some of the states were localized.

iii) It has been argued sometimes in the literature that the net effect of impurities could be an additional breaking of parity (and $T$ invariance) in the $2+1$ quantum system, triggering the emergence of a Chern-Simmons term in the effective action with a non standard coefficient. (The mass term itself breaks parity in $2+1$ dimensions.) We believe that the net effect of the impurities is simply to renormalize the fermion mass.

This work has been supported by the CICYT grant AEN89-0347 and the EEC Science Twinning Grant SCI-000337. J.M. acknowledges the financial support of a FPI graduate fellowship from the Ministerio de Educación y Ciencia.

\section{References}

[1] D.Espriu, M.Gross, P.Rakow and J.F.Wheater, Nucl. Phys. B275 (1986) 39; D.Espriu, M.Gross, P.Rakow and J.F.Wheater, Prog. Theor. Phys. (Supp.) 86 (1986) 304 See also: R.Friedberg, T.D.Lee and H.Ren, Prog. Theor. Phys. (Supp.) 86 (1986) 322; Y.Pang and H.Ren, Phys. Lett. B172 (1986); 195 (1987) 223; H.Ren, Nucl. Phys. B300 (1988) 531

[2] S.Coleman, Comm. Math. Phys. 31 (1973) 259

[3] S.Perantonis and J.F.Wheater, Nucl. Phys. B295 (1988)443

[4] H.B.Nielsen and N.Ninomiya, Nucl. Phys. B185 (1981)20; B193 (1981) 173

[5] W.Thirring, Ann. Phys. (N.Y.), 3 (1958) 91

[6] See e.g. R. Rajaraman, Phys. Rep. C5 (1975) 227, and references therein

[7] S.Coleman, Phys. Rev. D11 (1975) 2088

[8] R.F.Dashen, B.Hasslacher and A.Neveu, Phys. Rev. D11 (1975) 3424

[9] A.B.Zamolodchikov and Al.B.Zamolodchikov, Ann. Phys. (N.Y.) 120 (1979) 253

[10] H.Yokota, Prog. Theor. Phys. 77 (1987) 1450 F.Ruiz and R.F.Alvarez-Estrada, Phys. Lett. B 182 (1986) 354

[11] R.F.Dashen, B.Hasslacher and A.Neveu, Phys. Rev. D10 (1974) 4114 


\section{Figure Captions}

Fig. 1.- (a.) $\langle\bar{\psi} \psi\rangle$ as a function of the lagrangian mass for three volumes: $\left(V_{1}\right) 648,\left(V_{2}\right) 1656$ and $\left(V_{3}\right) 2304$. The density of impurities is $10 \%$. The dashed line is the value of $\langle\bar{\psi} \psi\rangle$ in a regular lattice. The value in the doped lattice is much bigger, particularly for small masses, suggesting that it is sensible to use the term "spontaneous symmetry breaking". The value of $\langle\bar{\psi} \psi\rangle$ is a decreasing function of the lattice size for a given mass. Antiperiodic b.c. have been used. (b.) Same as in (a), but for a fixed volume $(V=1656)$ and three densities of doping: $20 \%, 10 \%$ and $2,4 \% \cdot\langle\bar{\psi} \psi\rangle$ is closest to its regular lattice value for the smallest density of impurities. (c.) The value $\langle\bar{\psi} \psi\rangle$ predicted by sine-Gordon / Thirring (dashed line), including the fermionic and bosonic contributions, compared to the values obtained from the doped lattice (solid line). The size of the system is 1600 points and the doping density is $10 \%$. There are some uncertainties in the extraction of the masses from the propagators when $m \rightarrow 0$ which may change slightly the dashed line but the agreement is qualitatively good.

Fig. 2.- (a.) The fermion effective mass as a function of the lagrangian mass for a given density $(10 \%)$ and three volumes. For large lagrangian masses the effective mass $M$ is volume independent, but finite size effects are relevant for small masses. There is saturation to a finite value that decreases with increasing volume. (b.) The fermion effective mass $M$ for a given volume $(V=1600)$ and three densities of impurities. For large masses the growth of $M$ is linear in $m$ (dotted line), but with a coefficient that grows with the doping. The enlarged area shows the departure from the linear behaviour for small masses (finite size effects) shown in (a) for a $V=1024$ and different densities.

Fig. 3.- (a.) The scalar zero momentum propagator $P_{11}$ for $V=800,10 \%$ doping and $m=$ 0.45. The solid line is the doped lattice propagator, the crosses are the fit assuming that in the scalar channel there is simply a contribution from a fermion-antifermion pair with mass $2 M$ (twice the fermion effective mass) and nothing else. The wave function renormalization (which is different from one) is also taken from the fermion propagator. The fit is perfect. (b.) The pseudoscalar zero momentum propagator $P_{55}$ for $V=1600,10 \%$ doping and $m=0.15$. The dashed line is the fermion-antifermion contribution in a regular lattice with mass $2 M$. The difference between this and the doped lattice result (solid line) can be accounted for by a scalar particle (the Goldstone). The crosses are the combined fit.

Fig. 4.- (a.) $m_{55}$ for a fixed density (10\%) as a function of the lagrangian mass for three different volumes. For large masses the finite size effects are very small. (b.) Same as before, for a fixed volume $(V=1600)$ and three densities. The coefficient of the quadratic dependence on $m$ in $m_{55}^{2}$ clearly grows with the doping (solid line). The fit is extremely good even for very large masses. For small masses, as seen in (a), there are departures from this leading $m_{55} \sim m$ behaviour. 